1 Short title: Stromule formation is causally linked to ETI signaling

2 Author for contact: Martin Hartmut Schattat, martin.schattat@pflanzenphys.uni-halle.de, +49 3455526286

\title{
XopQ induced stromule formation in Nicotiana benthamiana is causally linked to ETI signaling and depends on ADR1 and NRG1
}

3 Jennifer Prautsch ${ }^{\mathrm{a}}$, Jessica Lee Erickson ${ }^{\mathrm{b}, \mathrm{c}}$, Sedef Özyürek $^{\mathrm{a}}$, Rahel Gormanns ${ }^{\mathrm{a}}$, Lars Franke ${ }^{\mathrm{c}}$, Jane E. Parker ${ }^{\mathrm{e}}$,

4 Johannes Stuttmann ${ }^{\text {b,f }}$, Martin Hartmut Schattat ${ }^{\mathrm{a}}$

$5 \quad{ }^{a}$ Biology, Plant Physiology, Martin-Luther-University Halle-Wittenberg, Halle, Germany

$6 \quad$ biology, Plant Genetics, Martin-Luther-University Halle-Wittenberg, Halle, Germany

$7 \quad$ CLeibniz-Institut for Plant Biochemistry, Halle, Germany

8 dBiochemistry and Biotechnology, Martin-Luther-University Halle-Wittenberg, Halle, Germany

$9 \quad{ }^{\mathrm{e}}$ Max Planck Institute for Plant Breeding Research, Cologne, Germany

10 Institute for Biosafety in Plant Biotechnology, Federal Research Centre for Cultivated Plants, Julius Kühn-

11 Institute (JKI), Quedlinburg, Germany

Author for contact: martin.schattat@pflanzenphys.uni-halle.de

The authors responsible for distribution of materials integral to the findings presented in this article in accordance with the policy described in the Instructions for Authors (www.plantphysiol.org) is Martin Hartmut Schattat (martin.schattat@pflanzenphys.uni-halle.de), Jane Parker (parker@mpipz.mpg.de) and Johannes Stuttmann (jstuttmann@gmail.com).

One sentence summary: Genetic analysis aligns effector triggered immunity (ETI) induced stromule formation with the ETI signaling cascade but not programmed cell death and questions stromule guided peri-nuclear plastid clustering.

This study was funded by the Deutsche Forschungsgemeinschaft (DFG, German Research Foundation) 400681449/GRK2498, STU642/1-1 and SFB-1403-414786233 as well as Martin-Luther-University core funding. Author Contributions: JLE, JS, JPa and MHS contributed to experimental design and the written text; JPr, SÖ, RG and LF conducted the A. tumefaciens inoculation experiments in N. benthamiana and respective image analysis; JLE conducted $X c v$ inoculation experiments and respective image analysis. 


\section{Abstract}

In Nicotiana benthamiana, expression of the Xanthomonas effector XopQ triggers ROQ1dependent ETI responses and in parallel accumulation of plastids around the nucleus and the formation of stromules. Both processes were proposed to contribute to ETI-related hypersensitive cell death and thereby to plant immunity. Whether these reactions are directly connected to ETI signaling events has not been tested. Here we utilized transient expression experiments to determine whether XopQ-mediated plastid reactions are a result of XopQ perception by ROQ1 or a consequence of XopQ virulence activity. We find that $N$. benthamiana mutants lacking ROQ1, both RNLs (NRG1 and ADR1) or EDS1, fail to elicit XopQ-dependent host cell death and stromule formation. Mutants lacking only NRG1 lost XopQ-dependent cell death but retained some stromule induction that was abolished in the RNL double mutant. This analysis aligns XopQ-induced stromules with the ETI signaling cascade but not to host programmed cell death. Furthermore, data reveal that XopQ-triggered plastid clustering is not strictly linked to stromule formation during ETI. Our data suggest that stromule formation, in contrast to chloroplast peri-nuclear dynamics, is an integral part of the $N$. benthamiana ETI response and that both RNL sub-types play a role in this ETI response. 


\section{Introduction}

Plastids exhibit exquisite developmental flexibility, as demonstrated by their capacity to differentiate into various plastid types with specialized functions, biochemical activities and internal structures, depending on the plant organ, developmental stage or environmental condition. Furthermore, plastids undergo extreme morphological changes, in some cases changing their shape within minutes or seconds (Gunning, 2005, Pyke, 2013, Delfosse et al., 2016). One highly dynamic feature of plastids is the projection of long, stroma-filled tubules formed by the two envelope membranes. These projections, also called stromules, are reliably observed when either the stroma or the envelope membranes are fluorescently labeled (Fig. 1; reviewed in Delfosse et al., 2016). Over the last two decades, stromules have been detected by fluorescence microscopy in an increasing number of plant species throughout the Viridiplantae ("green plants"; reviewed in Gray et al., 2001), suggesting that stromule formation emerged early during plant evolution. Examination of different plant tissues revealed that while stromule frequencies may vary, stromules are a ubiquitous feature of plastids (Köhler \& Hanson, 2000, Holzinger et al., 2008).

Stromules form in response to developmental cues and increase following exposure to various stresses or signaling molecules and metabolites connected to stress (Schattat \& Klösgen, 2011a, Gray et al., 2012, Mathur et al., 2012, Caplan et al., 2015, Vismans et al., 2016), suggesting stromule formation is strictly controlled by the plant. These observations led to the hypothesis that stromules participate in processes that are fundamentally important for plant survival during stress, to transmit signals and/or support physiological changes.

Despite their early emergence in the evolution of Viridiplantae and their frequent observation across tissues, our knowledge of stromule function is limited. To date, mutants with defects in signaling pathways regulating stromule formation were not identified. Therefore, it remains unclear which processes or functions are carried out by stromules during stress responses and how these might be executed. As an alternative to genetic dissection of stromule formation per se, we decided instead to test effects of mutants in defined stress responses for effects on stromule formation. Our aim is to gain insight into the role of stromules during adaptation to a specific stress and use genetic tools to decipher stromule function. 
bioRxiv preprint doi: https://doi.org/10.1101/2021.12.06.471425; this version posted December 11, 2021. The copyright holder for this preprint (which was not certified by peer review) is the author/funder, who has granted bioRxiv a license to display the preprint in perpetuity. It is made available under aCC-BY-NC-ND 4.0 International license.

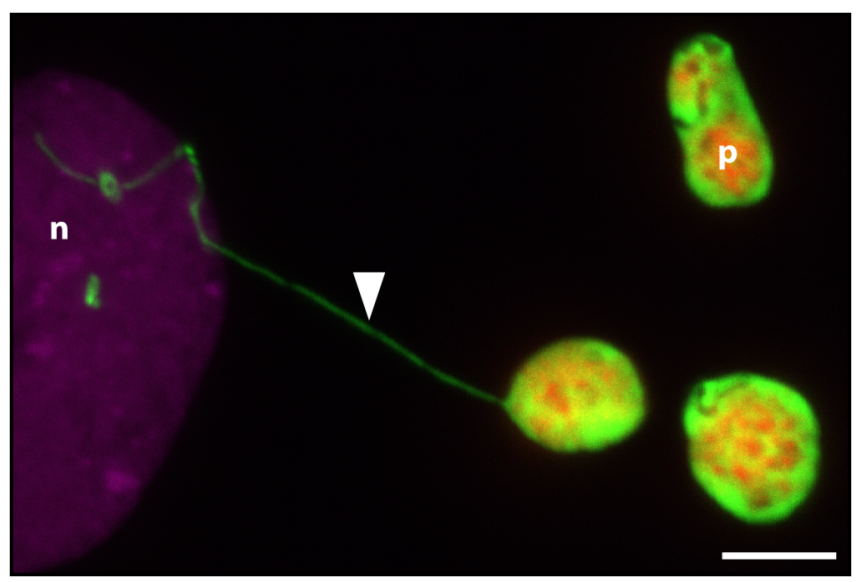

73 Fig. 1: Image of a stromule connecting a plastid with a nucleus in Nicotiana benthamiana.

74 CLSM fluorescence image of an $N$. benthamiana lower leaf pavement cell expressing a nuclear 75 marker (H2B:mCherry in magenta) and plastid stroma marker (FNR:EGFP in green); chlorophyll 76 autofluorescence is depicted in magenta; $\mathrm{p}=$ plastid body, $\mathrm{n}=$ nucleus, arrow $=$ stromule; scale bar corresponds to $5 \mu \mathrm{m}$ 
Biotic stress caused by plant interactions with recognized pathogens results in pronounced stromule formation (Krenz et al., 2012, Erickson et al., 2014, Caplan et al., 2015, Kumar et al., 2018, Adlung $\&$ Bonas, 2018). Many pathogenic microbes transfer virulence factors, known as effectors, into the host cell cytoplasm to promote infection, often by manipulating pattern-triggered immunity (PTI) programs induced by extracellular or intracellular immune receptors (Toruño et al., 2016, Büttner, 2016). In an incompatible interaction, intracellular immune receptors recognize one or more effectors. Effector recognition triggers a robust immune response termed effector-triggered immunity (ETI), which frequently culminates in localized host programmed cell death (a hypersensitive response $=\mathrm{HR}$ ) at infection sites (Cui et al., 2015, Yuan et al., 2021). Most intracellular immune receptors are nucleotide binding/leucine-rich repeat (NLR) proteins. NLR proteins are represented by two major pathogen-sensing NLR receptor classes, which are defined by their N/terminal domains. So called TIR-NLRs (TNLs) possess a Toll/interleukin-1 domain (TIR) and CC-NLRs (CNLs) a coiled-coil (CC) domain at their N-terminus. Additionally, different NLR protein families have been found to function in $A$. thaliana and Solanaceous species together with pathogen-detecting (sensor) NLRs in ETI, connecting sensor NLRs with downstream immunity factors and thus are called "helper" NLRs (Cui et al., 2015, Wu et al., 2019).

Dramatic increases in stromule frequencies were observed following expression of effectors recognized by CC-NLRs or TIR-NLRs prior to ETI-induced cell death in Nicotiana benthamiana and Arabidopsis thaliana (Caplan et al., 2015, Erickson et al., 2018). For example, induction of ETI (resulting in HR) via the transient co-expression of the p50 helicase domain from tobacco mosaic virus and the cognate TIR-NLR immune receptor, $\mathrm{N}$ from Nicotiana tabacum, in $N$. benthamiana results in strong stromule induction (Caplan et al., 2015). Similarly, a screen by our group revealed that expression of XopQ from the bacterium Xanthomonas campestris pv. vesicatoria ( $X c v$; strain 85-10), which is recognized by the TIR-NLR immune receptor RECOGNITION OF XopQ1 (ROQ1) in N. benthamiana, also strongly enhanced stromule frequencies (Schultink et al., 2017, Erickson et al., 2018). In the case of ETI activation via N/p50, the authors reported that many stromules were in close proximity to the nucleus, and appeared to make contact. This observation suggested that plastids might directly deliver defense signals to the nucleus via stromules (Caplan et al., 2015, Kumar et al., 2018). Stromule frequency also increased during CC-NLR and TIR-NLR mediated ETI in Arabidopsis thaliana when plants were challenged with avirulent strains of the bacterial pathogen Pseudomonas syringae (Caplan et al., 2015). Thus, it appears that stromule formation is a common response of plants during ETI.

In addition to an increase in stromule frequencies and stromule-to-nucleus contacts, formation of plastid clusters around nuclei was observed during ETI responses in N. benthamiana (Caplan et al., 
2015, Kumar et al., 2018), leading to the conclusion that plastid clusters might also support delivery of plastid-derived defense signals to the nucleus (Ding et al., 2019, Exposito-Rodriguez et al., 2020). In time lapse experiments spanning several minutes (Kumar et al., 2018), plastid bodies moved in the direction of stromule tips/anchor points in the majority of cases, giving the impression that stromules directionally pull the plastid body with them. This observation led to the conclusion that stromules might guide plastids to the nucleus to facilitate clustering. Hence, stromules near the nucleus might have a second function in plastid positioning.

Taken together, ETI-induced stromules present a starting point for more detailed genetic analyses of stromule formation and plastid clustering following the well-defined molecular event of effector recognition. For this, we chose $N$. benthamiana ROQ1-mediated XopQ recognition leading to ETI as a suitable system to critically examine the functional relationship between stromule formation and immunity signaling.

The TNL ROQ1 was reported to oligomerize, and assemble into a tetrameric holoenzyme with NADase activity (Ma et al., 2020, Martin et al., 2020), forming a structure similar to the pentameric complex reported for the $A$. thaliana CNL ZAR1 ('resistosome'), which may directly integrate into membranes to function as $\mathrm{Ca}^{2+}$-permeable channels (Wang et al., 2019a, b, Bi et al., 2021). ETI responses initiated by TNLs like ROQ1 require at least two more components: Heterodimeric complexes composed of the lipase-like protein ENHANCED DISEASE SUSCEPTIBILITY 1 (EDS1) and either PHYTOALEXIN DEFICIENT4 (PAD4) or SENSECENCE ASSOCIATED

132 GENE101 (SAG101), and two types of helper NLRs, which are characterized by an N-terminal four-helix bundle domain with homology to A. thaliana RESISTANCE to POWDERY MILDEW 8 (RPW8), and therefore are called RPW8-like coiled-coil $\left(\mathrm{CC}_{\mathrm{R}}\right)$ domain NLRs (RNLs) (Wagner et al., 2013, Jubic et al., 2019, Castel et al., 2019, Wu et al., 2019, Gantner et al., 2019, Lapin et al., 2019, Saile et al., 2020).

Molecular functions of EDS1 yet remain elusive, but its heterodimeric complexes are essential for TNL-mediated immune responses in different dicot plants including $A$. thaliana and $N$. benthamiana (Wagner et al., 2013, Gantner et al., 2019, Lapin et al., 2020). There are two types of RNLs known ACTIVATED DISEASE RESISTANCE GENE 1 (ADR1) and N REQUIREMENT GENE 1 (NRG1) and members of both types can be found in A. thaliana and N. benthamiana (Collier et al., 2011, Lapin et al., 2020). In A. thaliana, distinct EDS1-PAD4-ADR1 and EDS1SAG101-NRG1 modules appear to regulate pathogen resistance and cell death, respectively, in TNL immunity (Lapin et al., 2019, Sun et al., 2020). In contrast, immune functions are not known 
operate mainly through $N b$ NRG1 to mediate both cell death and resistance in this species (Qi et al., 2018, Gantner et al., 2019, Lapin et al., 2019). NbADR1 immune functions were so far not analyzed, but $\mathrm{Nb \_ nrgl}$ mutant plants, but not $\mathrm{Nb \_ eds1}$ mutant plants, still mobilize significant XopQ/Roq1-mediated transcriptional reprogramming, despite a loss of resistance and absence of cell death, thus suggesting some degree of cooperativity or redundancy between the two helper NLR classes also in N. benthamiana TNL immunity (Qi et al., 2018, Saile et al., 2020).

In this study, we capitalized on the previous characterization of XopQ/ROQ1-induced TNL immunity in $N$. benthamiana and positioned chloroplast stromule formation and perinuclear clustering in downstream signaling networks. Our data suggest that, although stromule formation is tightly linked to immune responses, it can be uncoupled from ETI-triggered cell death.

156 Furthermore, our data support partially redundant functions of the NRG1 and ADR1 helper NLRs (hNLRs) in stromule formation. Intriguingly, our data indicate that plastid clustering can be largely uncoupled from ROQ1 ETI and hence is unlikely to represent an integral component of the plant innate immune response.

\section{Results}

\section{$X c v$-mediated stromule formation in $N$. benthamiana depends on XopQ}

162

We previously reported that A. tumefaciens mediated transient expression of XopQ from Xcv induces stromule formation in N. benthamiana (Erickson et al., 2018). During infection with Xcv strains such as $X c v 85-10$, a strain which naturally delivers XopQ to host cells, XopQ is likely less abundant in infected cells than during transient expression experiments. Additionally, XopQ is translocated together with the entire type III-secreted effectome of $X c v$ ( $>30$ effectors; Teper et al., 2016). In order to test the extent to which stromule frequencies measured during transient expression experiments reflect the $X c v$ interaction, different bacterial strains were inoculated into FNR:eGFP expressing transgenic $N$. benthamiana plants. Under our greenhouse conditions the wild-type strain Xcv 85-10 induces an ETI-associated programmed cell death response (indicating XopQ recognition), showing first signs of dead leaf tissue at 2 dpi (Adlung et al., 2016). In order to be able to observe plastids in living cells, we collected leaf samples for microscopy 43 hours post-inoculation (hpi) (Fig. 2A). To test the role XopQ and other effectors play in stromule response, $X c v$ mutant strains $\Delta h r c N$ and $\triangle x o p Q$ were infiltrated on the same leaf with the wild-type strain. The $\Delta h r c N$ mutant is deficient in typeIII secretion and serves as a non-virulent PTI control (Lorenz \& Büttner, 2009). $\triangle h r c N$ as well as $\triangle x o p Q$ mutant strains of $X c v$ did not induce macroscopically visual changes in infected tissues at 2 dpi and were not distinguishable from the 
178 mock infiltration (Fig. 2A; Lorenz \& Büttner, 2009, Adlung et al., 2016, Adlung \& Bonas, 2017).

179 When analyzing the stromule phenotype, treatments differed significantly: The $X c v$ 85-10 strain

180 induced massive stromule induction in the infiltrated tissue. The $\triangle x o p Q$ and $\Delta$ harcN (no effector

181 translation) mutant-inoculated tissue harbored almost no stromules, with levels comparable to mock

182 inoculations $(10 \mathrm{mM} \mathrm{MgCl}$ ) and $\Delta h r c N$, which does not translocate any effectors (Fig. 2B,

183 Supplemental materials Table S1).

184 Together with transient expression experiments using A. tumefaciens (Erickson et al., 2018), these

185 results indicate that stromule induction at 2 dpi by the wild-type strain is strictly dependent on

186 presence of XopQ. No other effectors in this strain contributed measurably to stromule induction

187 at $2 \mathrm{dpi}$, when translocated at natural levels. In conclusion, inoculations with $X c v$ strains show that

188 XopQ-triggered stromules appear during pathogen attack at 2 dpi, and supports the idea that

189 stromule induction during transient assays recapitulates a physiologically relevant phenotype. 


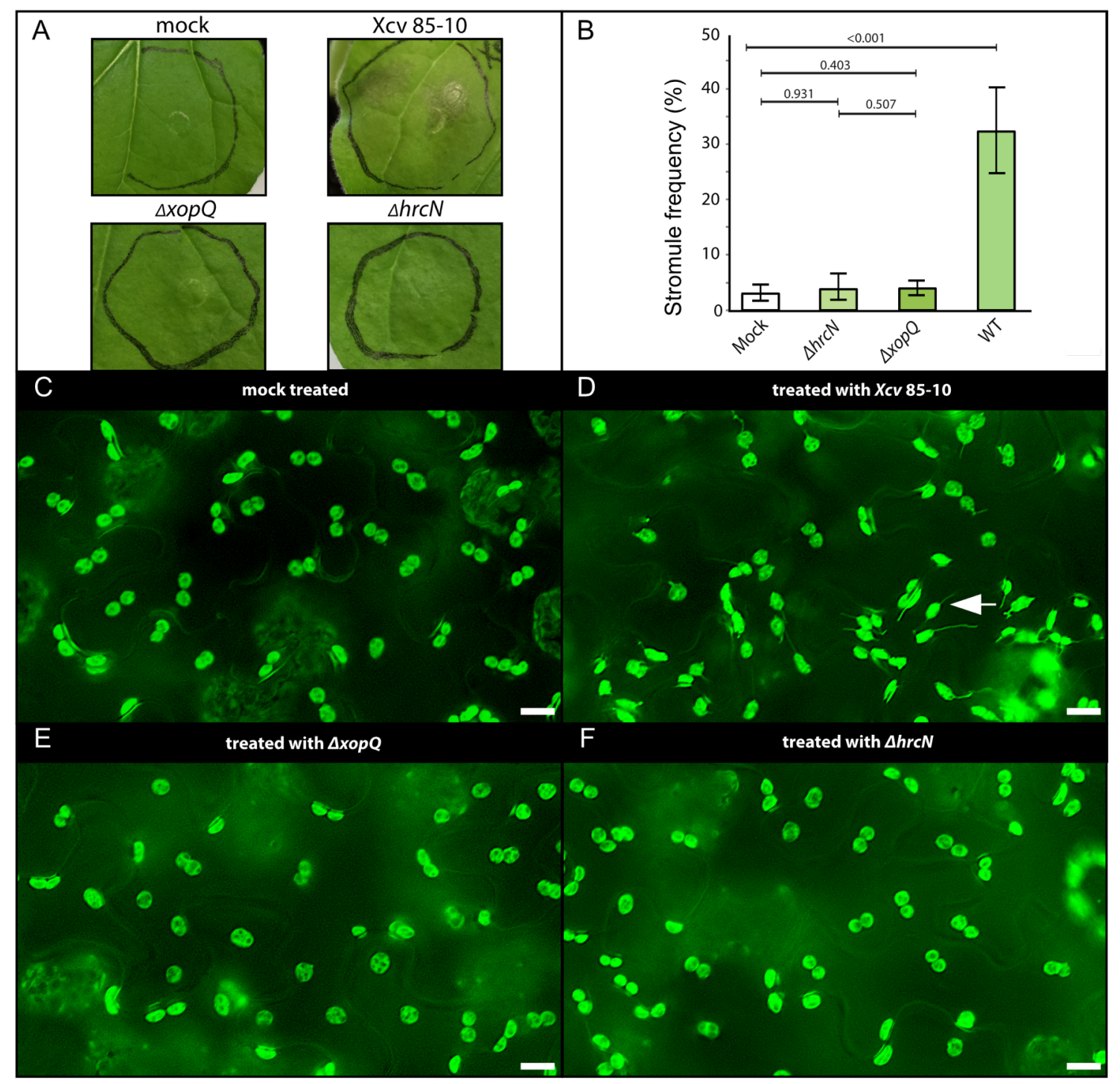

192 Fig. 2: Xanthomonas campestris pv. vesicatoria (Xcv) inoculation experiments in wild-type

193 FNR:eGFP transgenic plants. (A) Macroscopic phenotypes of leaves infiltrated with different

194 Xcv strains at 2 dpi.(B) Stromule frequency (SF\%) of Xcv-inoculated tissue at 43 hpi. Bars in the

195 bar plot represent arithmetic averages (three repeats with 5 plants each); error bars represent 95\%

196 confidence intervals; p-values of statistical tests are shown above the black lines; (C - F) Sample

197 sectors of representative microscopic images used for stromule quantification. Green fluorescence

198 originates from the stably expressed FNR:eGFP plastid stroma marker; scale bars $=10 \mu \mathrm{m}$;

199 arrow $=$ stromule. (full-frame images Supplemental materials Fig. S1 and S2) 


\section{XopQ fails to induce high stromule frequency values in roq1 mutant plants}

202

203

204

205

206

207

208

We next examined the extent to which XopQ-mediated stromule induction in the lower epidermis of $N$. benthamiana is a consequence of XopQ perception by TNL receptor ROQ1, using $A$. tumefaciens-based transient gene expression. Different $N$. benthamiana mutant lines impaired in XopQ perception or lacking TNL downstream signaling components were co-infiltrated with Agrobacterium strains for expression of stroma-targeted eGFP (SSU:eGFP) and either xopQ:mOrange 2 or mOrange 2 alone, as control. Agrobacterium strains were infiltrated at a final $\mathrm{OD}_{600}=0.2$, which led to only moderate Agrobacterium-dependent stromule induction $(<20 \%$, Erickson et al., 2014) well below XopQ-induced stromule frequencies $(\sim 60 \%)$. These experimental conditions were evaluated using $N$. benthamiana stably expressing stromal-targeted eGFP (FNR:eGFP; Supplemental materials Fig. S3).

We first tested XopQ-mediated stromule frequencies in roq1 mutant plants (roq1-3 and -4). ROQ1deficient plants fail to recognize XopQ and therefore lack the typical ETI-induced yellowing and chlorosis exhibited by wild-type plants following transient XopQ expression (Schultink et al., 2017; Gantner et al., 2019). xopQ:mOrange 2 or the mOrange 2 control were co-infiltrated with a stromatargeted GFP (ssu:eGFP) into wild-type and mutant plants to allow for the visualization of plastids and stromules. As a control for XopQ recognition, macroscopic phenotypes of the co-infiltrated leaves were recorded $10 \mathrm{dpi}$, a time point when symptoms are clear despite the low optical densities used for infiltration resulted (Fig. 3A). As expected, xopQ:mOrange2 expression in wild-type plants resulted in chlorosis of the infiltration spot, indicative of the XopQ-induced ETI response (Adlung et al., 2016). In roq 1-3 and roq 1-4 plants, there was no visible chlorosis and tissues were indistinguishable from control infiltrations. In all plant lines, mOrange 2 controls showed stromule frequency values characteristic for leaves infiltrated with „empty“ GV3101 (pMP90) bacteria (compare Fig. 3B with Supplemental materials Fig. S3), indicating that the roq1 mutation does not increase basal stromule frequencies. xopQ:mOrange2 expression resulted in significantly higher stromule induction in wild-type plants, as previously described (Erickson et al., 2018). In contrast, xopQ:mOrange2 expression failed to induce stromules beyond GV3101 (pMP90) basal levels in the roq1-3 and roq1-4 mutant plants (Fig. 3B; Supplemental materials Table S2). Average stromule frequencies in mutants infiltrated with xopQ:mOrange 2 were equal to or less than mORANGE2 controls. Fig. 3C, D and f show representative microscopy images of xopQ:mOrange2 expressing tissues. The full loss of XopQ-induced stromule formation in roq1 mutant plants shows that XopQ recognition by ROQ1 is required for stromule induction, and that non-recognized XopQ activity does not generate a stromule-inducing signal. 
bioRxiv preprint doi: https://doi.org/10.1101/2021.12.06.471425; this version posted December 11,2021 . The copyright holder for this

preprint (which was not certified by peer review) is the author/funder, who has granted bioRxiv a license to display the preprint in perpetuity. It is made available under aCC-BY-NC-ND 4.0 International license. 


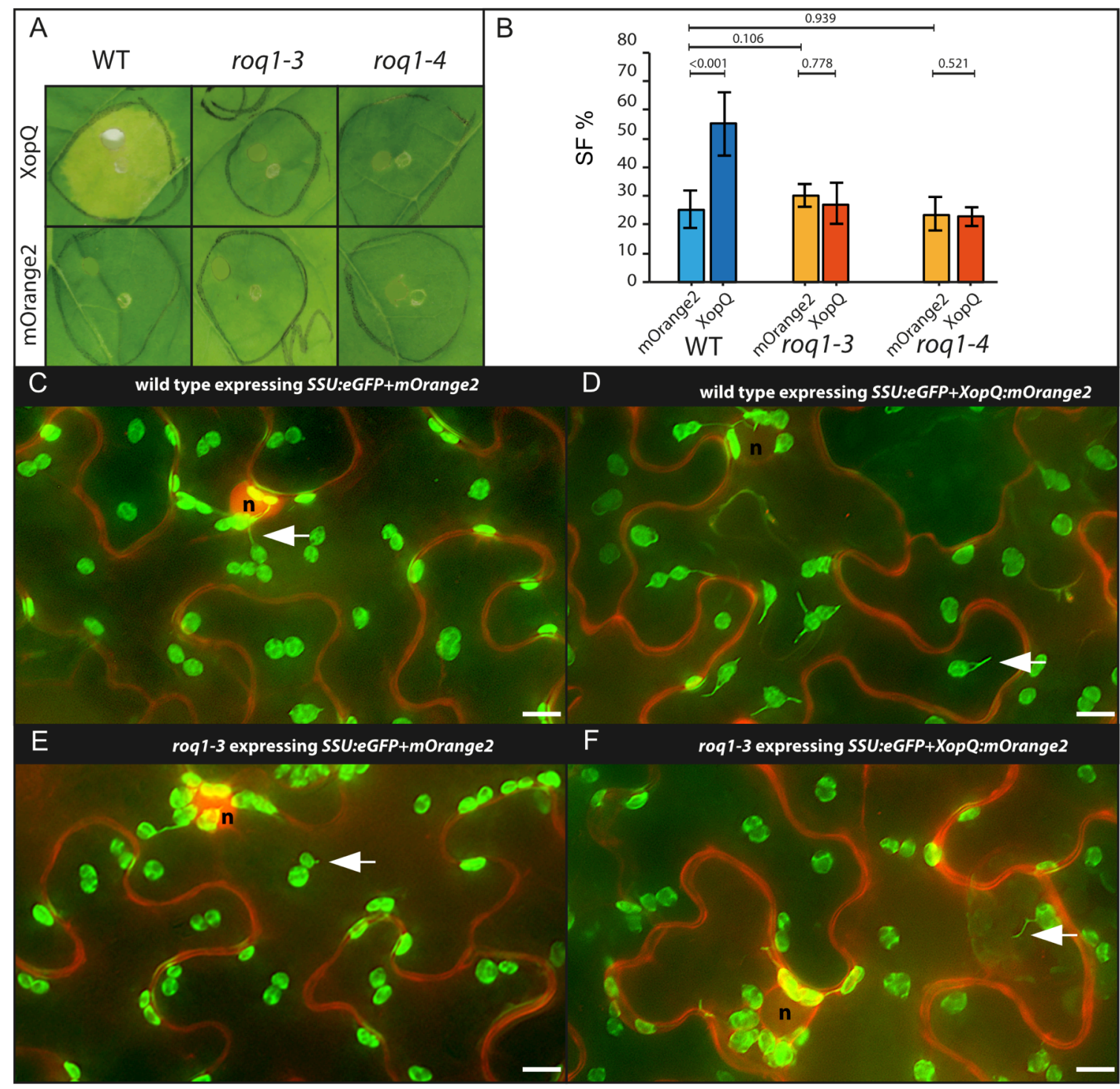

236 Fig. 3: Test for XopQ mediated stromule formation in lower leaf epidermis cells of roq1

237 mutants (Nicotiana benthamiana). (A) Macroscopic phenotypes of Agrobacterium-mediated

238 xopQ:mOrange2 and mOrange2-expression in wildtype, roq1-3 and roq1-4 leaves 10 dpi. (B)

239 Results of stromule quantification expressed as stromule frequency (SF\%). Bars in the bar plot

240 represent arithmetic averages; error bars represent 95\% confidence intervals; $p$-values of the

241 statistical tests are shown above the black lines. (C - F) Sample sectors of representative

242 microscopic images used for stromule quantification. Green fluorescence originates from the

243 SSU:eGFP plastid stroma marker; red fluorescence originates from the mORANGE2

244 fluorescence protein ( $\mathrm{c}$ and $\mathrm{e}=$ mOrange 2 controls; $\mathrm{d}$ and $\mathrm{f} m$ Orange 2 fused to $x o p Q$ ); nuclei $=$ 245 "n"; arrow = stromule; scale bars $=10 \mu \mathrm{m}$. (full-frame images Supplemental materials Fig. S4 246 and Fig. S5) 


\section{XopQ/ROQ1-dependent stromule induction requires EDS1}

249 EDS1 is essential for resistance and cell death mediated by TNL-type immune receptor ROQ1

250 (Adlung et al., 2016, Gantner et al., 2019). In order to test if XopQ-mediated stromule formation 251 is dependent on EDS1, mixed infiltrations were repeated in eds 1 a-1 knockout and wild-type plants. 252 In respect to stromule frequency and macroscopic phenotype the wildtype plants responded as seen 253 in previous experiments (Fig. 4A and B; Supplemental materials Table S2). In contrast to the 254 wildtype eds $1 a-1$ plants did not show signs of chlorosis at $10 \mathrm{dpi}$ in response to xopQ:mOrange2 255 expression, which is consistent with literature reports (Fig. 4A; Adlung et al., 2016, Gantner et al., 256 2019). As was the case for roq1 mutant plants, XopQ-induced stromules were not observed in 257 eds $1 a-1$ tissues (Fig. 4B and F; Supplemental materials Table S2). These results indicate that 258 stromule formation in response to XopQ occurs downstream of EDS1 signaling, suggesting that 259 XopQ-ROQ1 interaction and ROQ1 tetramerization ('resistosome' formation; Schultink et al., 260 2017, Martin et al., 2020) are not sufficient to induce stromules. 


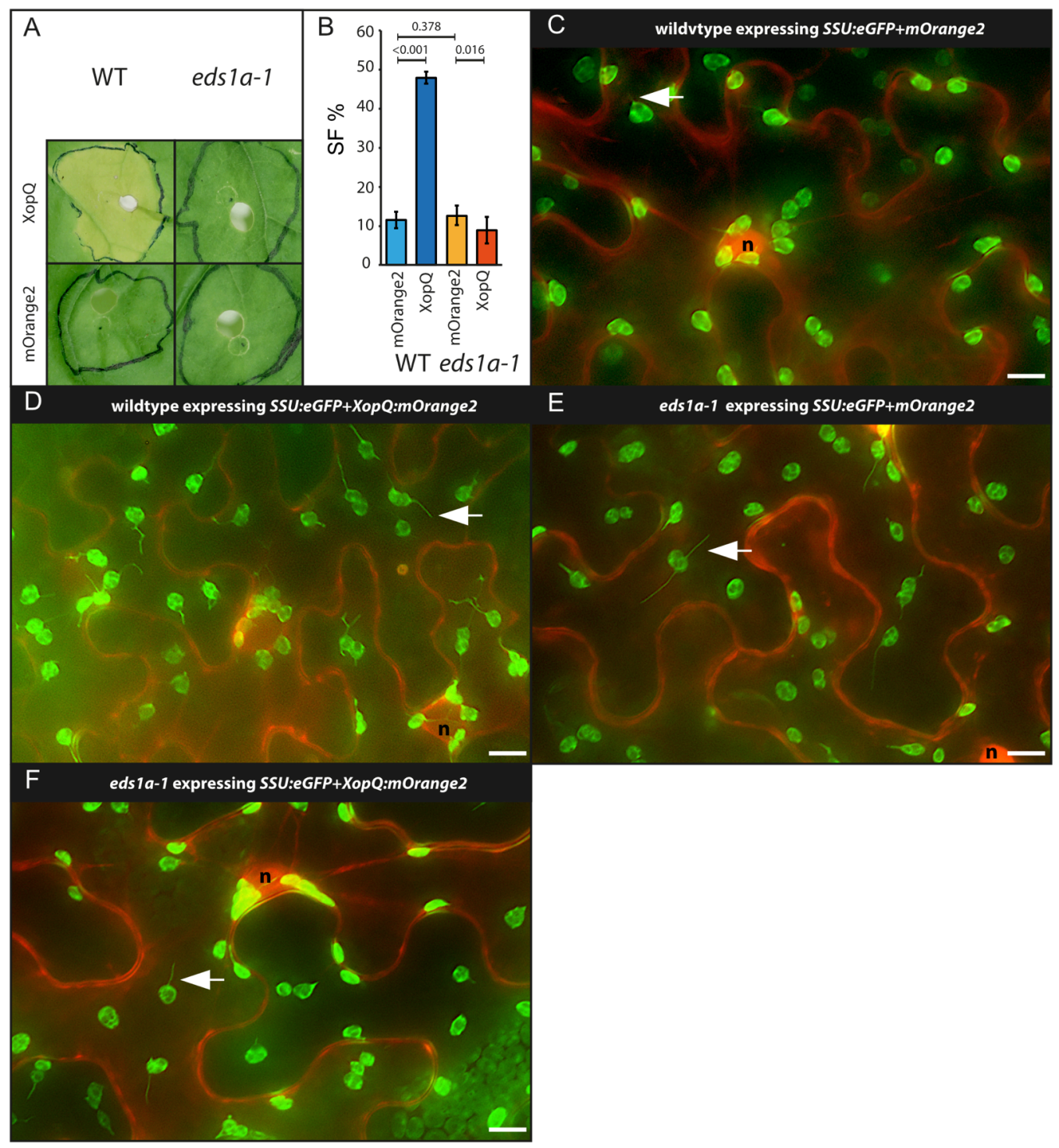

263 Fig. 4: Test for XopQ-mediated stromule formation in the lower leaf epidermis of eds1a-1

264 mutants (Nicotiana benthamiana). (A) Macroscopic phenotypes of xopQ:mOrange2 and

265 mOrange 2 infiltrated wildtype and eds $1 a-1$ leaves 10 dpi. (B) Results of stromule quantification

266 expressed as stromule frequency (SF\%). Bars in the bar plot represent arithmetic averages (three 267 repeats with 5 plants each); error bars represent 95\% confidence intervals; $p$-values of the 268 statistical tests are shown above the black lines; (C - F) sample sectors of representative 269 microscopic images from the data set used for stromule quantification. Green fluorescence 270 originates from the SSU:eGFP plastid stroma marker; red fluorescence originates from the 271 mORANGE2 fluorescence protein (c and e untagged mOrange2; $d$ and $f$ mOrange 2 fused to 272 XopQ); nuclei = " $n$ "; arrow = stromule; scale bars $=10 \mu \mathrm{m}$. (full-frame images Supplemental 273 materials Fig. S6.) 

cell death

In A. thaliana, RNL-type NLRs of the ADR1 and NRG1 subfamilies contribute to TNL immunity (Castel et al., 2019, Wu et al., 2019, Lapin et al., 2019, Saile et al., 2020). In N. benthamiana nrgl mutant plants, resistance and cell death induced by several TNLs was fully abolished, suggesting NRG1 as the major RNL in TNL immunity in this species (Qi et al., 2018). To test if XopQstimulated stromule formation is NRG1-dependent, two mutant lines with different genomic deletions, $n r g 1-4$ and nrgl-5 (Ordon et al., 2021) were analyzed. The $n r g 1$ mutants did not show signs of yellowing in response to A. tumefaciens mediated XopQ expression, and infiltration spots were macroscopically indistinguishable from control infiltrations (Fig. 5A), as expected (Qi et al., 2018, Ordon et al., 2021). All three plant lines responded similarly to the mOrange 2 control expression, with stromule frequencies reaching approximately $25 \%$ (Fig. 5B; Supplemental materials Table S2). In contrast, the response to xopQ:mOrange2 was markedly different between wild-type and $n r g 1$ mutant lines (Fig. 5B). xopQ:mOrange 2 expression in the $n r g 1$ background induced stromule frequencies values, which were between Agrobacterium-inoculated and xopQ:mOrange 2 -inoculated wild-type plants. Although $r o q 1$, eds 1 and $n r g 1$ mutants were equally deficient in XopQ-induced cell death (necrosis), stromule formation did not strictly require NRG1, and is thus uncoupled from cell death in $N b \_n r g l$ mutant plants.

So far, a function of ADR1 was not identified in N. benthamiana (Lapin et al., 2019, Sun et al., 2021) but varied contributions of these RNLs to Arabidopsis TNL ETI suggested that $N$. benthamiana ADR1 might steer residual stromule formation in N. benthamiana nrgl lines. Therefore, we tested stromule formation in response to XopQ in a newly generated Nbadrl_nrgl double mutant line using mixed infiltrations as before. As in the nrgl single mutants, xopQ:mOrange 2 expression did not induce yellowing or cell death in $a d r 1 \_n r g 1$ plants (Fig. 6A). In contrast to our observations in $n r g l$ single mutants, xopQ:mOrange 2 did not induce the formation of stromules beyond the mORANGE2 control (Fig. 6B; Supplemental materials Table the roql (Fig. 3A) and eds 1 (Fig. 4A) mutant lines. Overall, these results show that not only NRG1 but also ADR1 contributes to stromule formation in $N$. benthamiana. Hence, these results uncover that the ADR1-family of RNLs exhibits not only ETI signaling functions in A. thaliana ETI but also functions in the ETI response of solanaceous plants. 


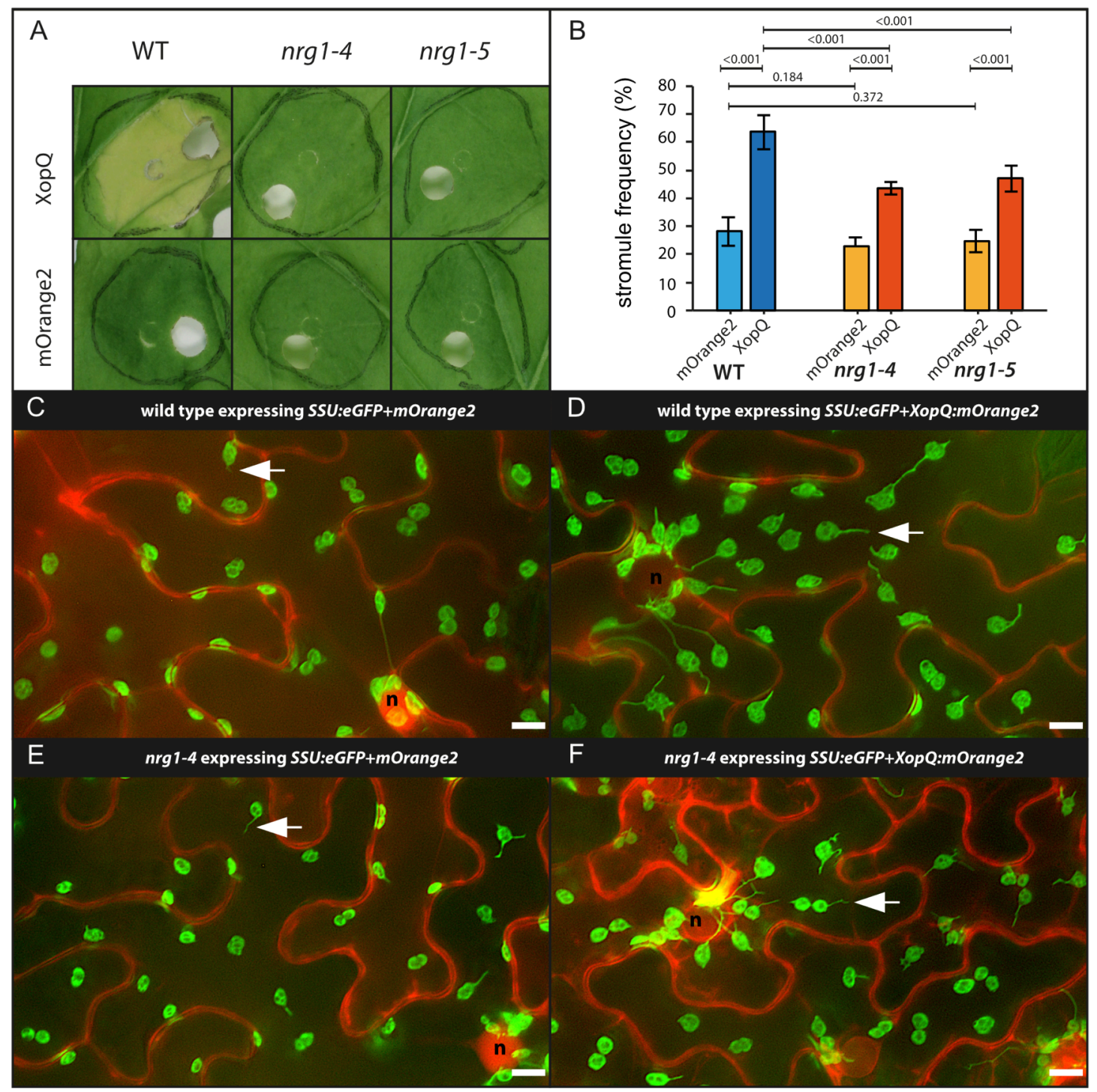

Fig. 5: Test for XopQ mediated stromule formation in lower leaf epidermis cells of $\boldsymbol{n r g} 1$

mutants (Nicotiana benthamiana). (A) Macroscopic phenotypes of XopQ:mOrange2 and mOrange2-infiltrated wildtype, $n r g 1-4$ and $n r g 1-5$ leaves at 10 dpi.(B) Results of stromule quantification expressed as stromule frequency (SF\%). Bars in the bar plot represent arithmetic averages (three repeats with 5 plants each); error bars represent $95 \%$ confidence intervals; $p$-values

313 of the statistical tests are shown above the black lines. $(\mathbf{C}-\mathbf{F})$ Sample sectors of representative 314 microscopic images used for stromule quantification. Green fluorescence originates from the 315 SSU:eGFP plastid stroma marker; red fluorescence originates from the mORANGE2 fluorescence 316 protein ( $\mathrm{c}$ and $\mathrm{e}=$ Mrange 2 control; $\mathrm{d}$ and $\mathrm{f}=$ Orange 2 fused to $X o p Q$ ); nuclei $=$ " $\mathrm{n}$ "; arrow = 317 stromule; scale bars $=10 \mu \mathrm{m}$. (full-frame images in Supplemental materials Fig. S7). 

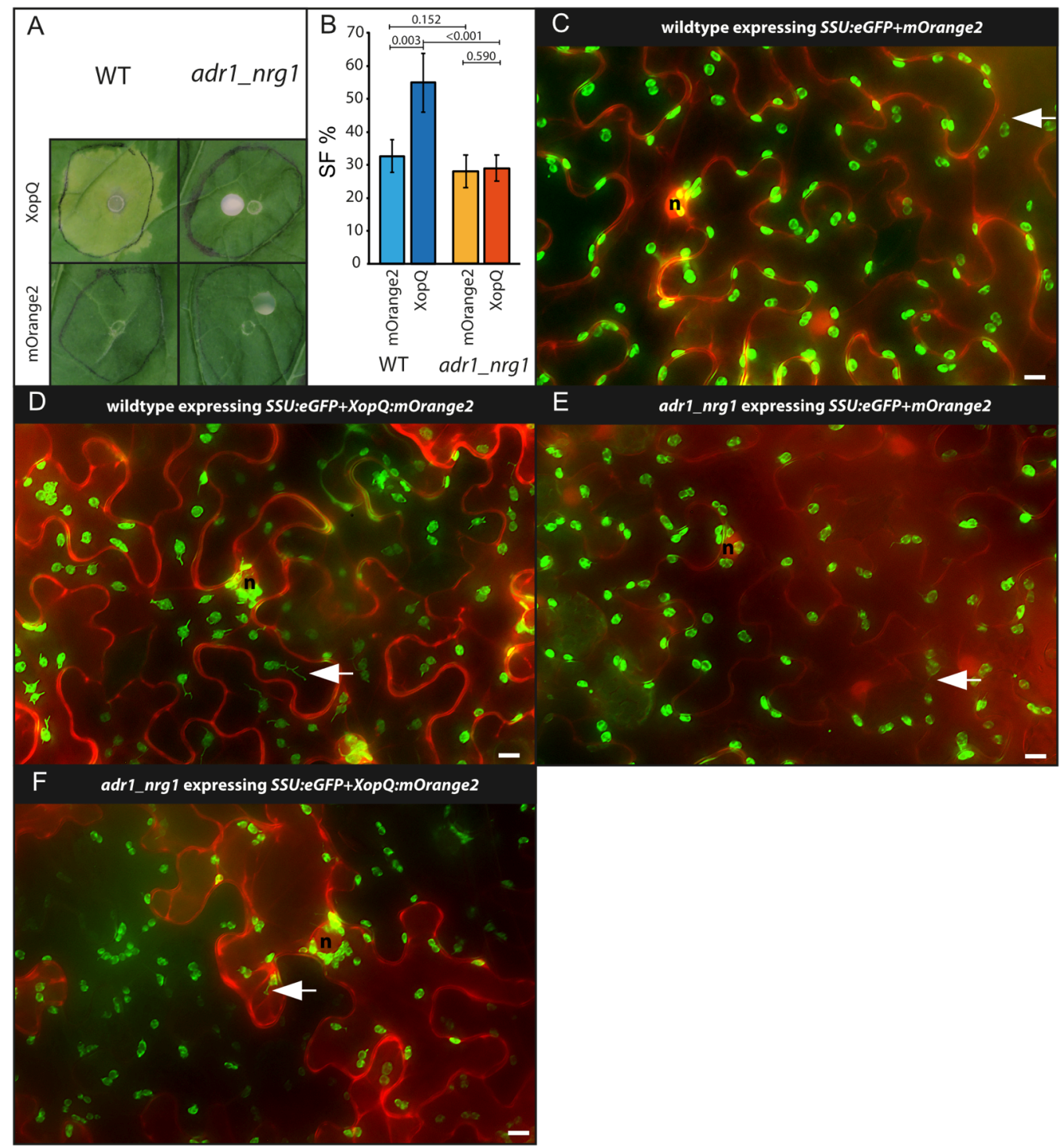

320 Fig. 6: Test for XopQ mediated stromule formation in lower leaf epidermis cells of

321 adr1_nrg1 double mutants (Nicotiana benthamiana). (A) Macroscopic phenotypes of

322 xopQ:mOrange2 and mOrange2-infiltrated wildtype and $a d r 1 \_n r g 1-5$ leaves at 10 dpi. (B)

323 Results of stromule quantification expressed as stromule frequency (SF\%). Bars in the bar plot

324 represent arithmetic averages; error bars represent 95\% confidence intervals; $p$-values of the

325 statistical tests are shown above black lines; wild-type 3 plants and adrl_nrgl 5 plants for each of

3263 repeats. (C - F) Cropped images used for stromule quantification. Green fluorescence originates

327 from the SSU:eGFP plastid stroma marker; red fluorescence originates from the mORANGE2

328 fluorescence protein (c and e are mOrange 2 controls; $\mathrm{d}$ and $\mathrm{f}$ show mOrange 2 translation fusion

329 with $X o p Q$ ); nuclei = "n"; arrow = stromule; scale bars $=10 \mu \mathrm{m}$. (full-frame images shown in

330 Supplemental materials Fig. S8). 


\section{XopQ-induced peri-nuclear plastid clustering does not require TNL immune signaling}

333 In order to test if XopQ-induced ETI facilitates formation of chloroplast clusters, and whether this

334 has the same genetic dependencies as found for stromule frequency, chloroplast clustering was 335 quantified in wild-type, roq1-3, eds1a-1, nrgl-4 and adrl_nrgl lines. As a measure for plastid 336 clustering, the number of plastids in close proximity (up to one plastid in diameter) to the nucleus 337 was counted and expressed as the plastid nucleus association index (PNAI, see Erickson et al., 338 2014, 2018). In these experiments, mORANGE2 or XopQ:mORANGE2 fluorescence, 339 respectively, served to highlight the position of nuclei (see Fig. 7B - K). In control infiltrations 340 (mOrange2), wild-type and all four mutants produced similar numbers of plastids around the 341 nucleus (Fig. 7A; Supplemental materials Table S3). No significant differences in PNAI were 342 detected. When challenged with XopQ, plastid clustering increased in wild-type plants (Fig. 7A; 343 Supplemental materials Table S3). Although plastid clustering has been considered an ETI response 344 (Caplan et al., 2015, Kumar et al., 2018, Ding et al., 2019), we found that XopQ-induced plastid 345 clustering was not diminished in mutant lines impaired in XopQ recognition or downstream 346 signaling (Fig. 7A; Supplemental materials Table S3). Upon expression of xopQ:mOrange2, nrg13474 showed wild-type levels of plastid clustering, while roq1-3, eds 1 and adr1_nrgl mutants had 348 significantly higher PNAI values (Fig. 7A; Supplemental materials Table S3). Fig. 7B - K shows 349 representative images of the different plant lines expressing ssu:eGFP+xopQ:mOrange2 or 350 ssu:eGFP+mOrange2.

351 We concluded that when the XopQ-induced ETI signal cascade is blocked (roql, eds 1 and 352 adrl_nrgl mutants), the tendency of plastids to cluster around the nucleus remains, and is even 353 enhanced compared to wild-type or plants ( $\mathrm{rgl}$ mutant) with partially intact signaling (Fig. 7H). 354 These data suggest that one feature of ROQ1/XopQ-induced ETI is suppression of plastid 355 clustering. 


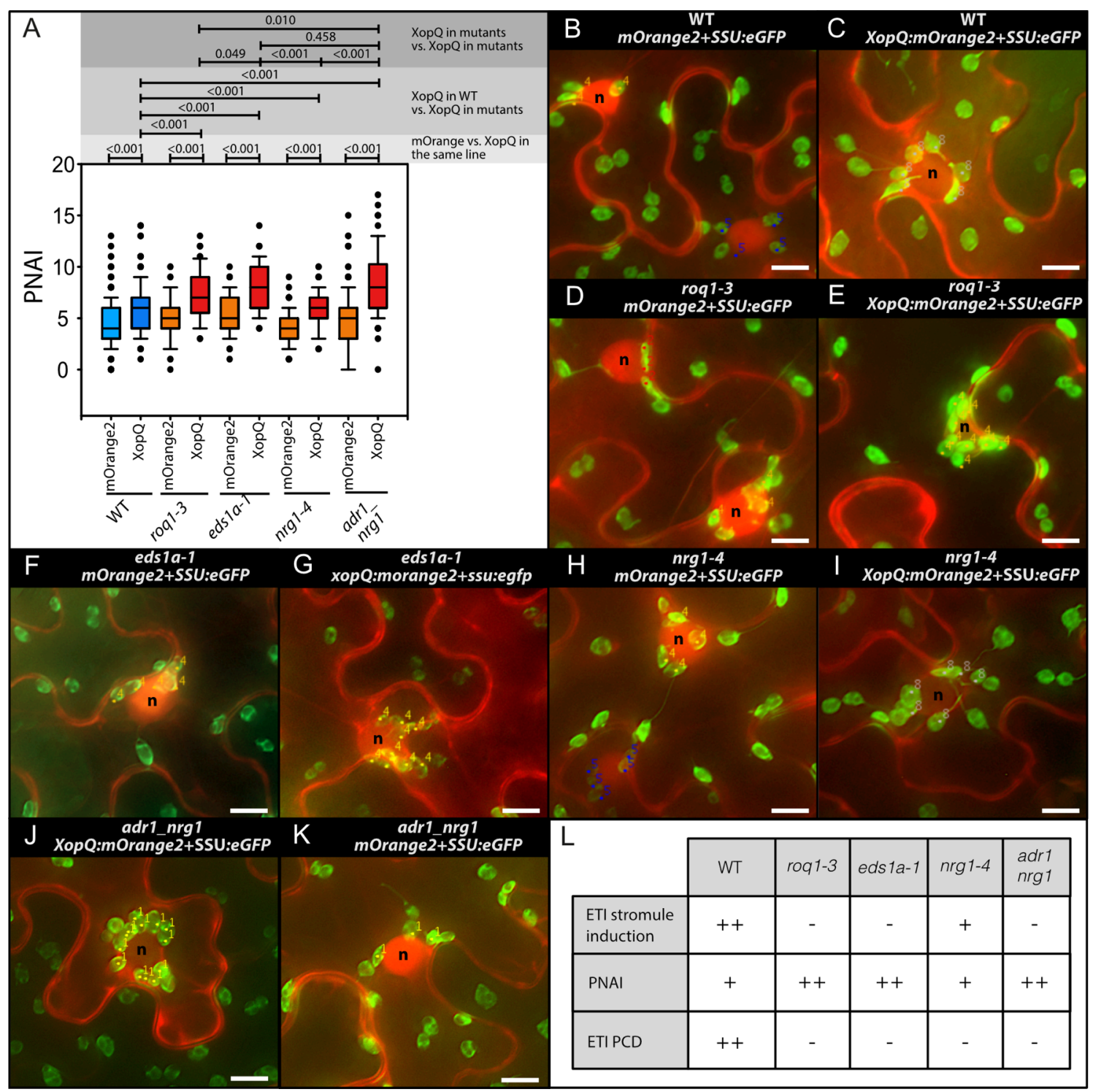

359 benthamiana). Plastid nucleus association index (PNAI) at 3 dpi following mOrange2 and 360 xopQ:mOrange2 expression in wild/type as well as mutant plant lines (roq1-3, eds 1a, nrg1-4 and $361 a d r 1 \_n r g 1$ ). (A) PNAI values represented in box plots (box line $=$ median, whiskers $=10^{\text {th }}$ as well 362 as $90^{\text {th }}$ percentile, with each outlier plotted); p-values of Mann-Whitney rank sum tests are shown 363 above the lines in grey boxes. (B - K) Sample images of nuclei with associated plastids (labeled 364 with a dot a number as they were during original plastid scoring); " $\mathrm{n}$ " = nuclei; scale bars $=10 \mu \mathrm{m}$.

365 (L) Summary of observed ETI stromule, PNAI and cell death phenotypes where "-." = no change 366 compared to control, “+” = visible but moderate increase in cell death; "++" = strong increase. 


\section{Discussion}

Here, we set out to understand how ETI-associated stromule formation aligns with signaling processes downstream of immune receptor activation, using recognition of the effector XopQ by the TNL ROQ1 in N. benthamiana as a case study. A quantitative analysis of stromule formation and peri-nuclear plastid clustering in XopQ recognition and ETI signaling mutants produced several important insights. First, complete absence of a XopQ-related stromule response in roq1 and eds 1 mutants shows that XopQ-induced stromules are not a result of its virulence/effector activity, but result from effector recognition by the ROQ1 immune receptor and a resulting EDS1-dependent ETI response. Second, residual induction of stromules in the $n \mathrm{rgl}$ mutant in the absence of appreciable cell death suggests that induced stromule formation is not a consequence of ETI-related host cell death but more closely related to pathogen resistance. Third, analysis of the nrgl_adrl double mutant line reveals that residual XopQ-induced ETI and stromule frequency in $n r g l$ (but not eds1), is conferred by ADR1 in N. benthamiana. This reveals an ADR1 contribution to TNL ETI processes in a solanaceous plant, suggesting usage of both RNLs NRG1 and ADR1 branches in ETI, as observed in Arabidopsis. Finally, ROQ1/XopQ-induced plastid clustering does not correlate with ETI induction and stromule formation and is thus likely to be a direct or indirect consequence of XopQ virulence activity during infection. A model summarizing these findings is presented in Fig. 8A and B.

\section{Uncoupling immune signaling and HR-induced stromules}

Stromules have been proposed to transmit retrograde signals to the nucleus, and amplify programmed cell death responses as part of ETI (Caplan et al., 2015). More recently it was suggested that stromules have the added function of locating and pulling plastid bodies to the nucleus (Kumar et al., 2018). So far, all reported microbial effectors that induce stromules also provoked programmed cell death (Caplan et al., 2015, Erickson et al., 2018). Therefore, it remained unclear whether stromule formation accompanies the cascade of events contributing to host cell death or is a by-product of physiological changes in cells as they die. The observed stromule induction upon XopQ expression in $\mathrm{nrgl}$ mutant plants without cell death or measurable resistance (Qi et al., 2018) (Fig. 5A) suggests stromules represent events upstream of ETI-related pathogen resistance and host cell death. Hence, ETI-induced stromule formation is not coupled to cellular destruction but more likely an integral part of the ETI response, as suggested previously (Caplan et al., 2015). 
400

401

402

403

404

405

406

407

408

409

410

411

412

413

414

415

416

417

418

419

420

421

422

423

424

Dicot genomes encoding TNL receptors generally also encode RNL-type NLRs of the ADR1 and NRG1 classes (Collier et al., 2011, Lapin et al., 2020). In A. thaliana, both classes of RNLs contribute to immunity to different extents. Three $A$. thaliana ADR1 paralogs have functions in basal immunity to virulent pathogens related to salicylic acid, and they also contribute to PTI (Bonardi et al., 2011, Jubic et al., 2019, Pruitt et al., 2021, Tian et al., 2021). ADR1s and NRG1s contribute to different extents to A. thaliana TNL immunity, and function as distinct modules, respectively with EDS1-PAD4 and EDS1-SAG101 dimers regulating pathogen resistance and cell death (Lapin et al., 2019, Saile et al., 2020, Lapin et al., 2020, Sun et al., 2021). In N. benthamiana, only EDS1-SAG101 appeared to execute TNL ETI, and NRG1 was identified as a major RNL required for cell death and resistance mediated by several tested TNLs (Qi et al., 2018, Gantner et al., 2019). A role for $N$. benthamiana ADR1 in immunity was so far not detected, although Qi et al. (2018) reported residual transcriptional reprogramming occurring upon ROQ1 activation in $n r g 1$, but not eds 1 mutant plants. Notably, NbADR1 was among the upregulated genes of an NRG1independent regulon (Qi et al, 2018). Residual stromule formation in $n r g l$, but not edsl or nrg1_adr1 (compare panel '(A)' in Supplemental materials Fig. S4, 5 and 6) supports an NbADR1 contribution to TNL-ETI in N. benthamiana. Hence, stromule formation appears to be a highly sensitive read-out for ETI induction, occurring in the absence of cell death or measurable pathogen resistance which are blocked in $N$. benthamiana $n r g l$ and eds 1 mutants (Qi et al 2018). Current evidence suggests that, besides the sensor CNL ZAR1, RNLs also might assemble into poreforming resistosome complexes and function as $\mathrm{Ca}^{2+}$ permeable cation channels (Wang et al., 2019a, Jacob et al., 2021, Bi et al., 2021). Induced $\mathrm{Ca}^{2+}$ influx into host cells would then amplify ROS generation and salycilic acid signaling as well as transcription ( $\mathrm{Lu}$ and Tsuda 2021, Yuan et $a l ., 2021)$. In future work, it will be interesting to examine whether $\mathrm{Ca}^{2+}$ levels inside cells influence stromule formation, as stromule-to-nucleus connections were found to contribute to ROS formation (Caplan et al., 2015).

\section{Is perinuclear plastid clustering independent of ETI stromule induction?}

In an emerging concept, plastids are the source of important immune response signaling and defense metabolites, including precursors of salicylic acid and jasmonic acid. Many plastid-derived signals must reach the nucleus to fulfill their proposed functions (reviewed in Kretschmer et al., 2020). Thus, re-localization of plastids towards the nucleus might promote more efficient signal transmission. Indeed, when challenged with different pathogens and $\mathrm{H}_{2} \mathrm{O}_{2}$, plastids relocated towards the nucleus in $N$. benthamiana epidermis leaf cells, forming peri-nuclear clusters (Erickson et al., 2014, Caplan et al., 2015, Ding et al., 2019a). How plant cells regulate re-localization of plastids to the nucleus upon different stimuli remains unknown. Based on observations of stromule 
orientation often coinciding with plastid directional movement, it was proposed that stromules are initiated during ETI and extended along the microtubules network, finding anchor points on actin filaments close to the nucleus which guide plastid body movement towards nuclei (Kumar et al., 2018). Peri-nuclear plastid clustering, as a consequence, might enhance plastid-to-nucleus signal transfer underpinning immune responses. When we challenged wild-type plants with XopQ:mOrange2, stromule formation as well as perinuclear plastid clustering were consistently induced in lower epidermis cells (Fig. 7A -K). Additionally, stromules facing the nucleus and seemingly anchored in the nuclear periphery were observed (see Fig.s 3B, 4B, 5B and 6B for SF\%; e.g. 3D, 4D and 5D for nucleus-associated stromules). Both observations support stromules guiding plastid body movement (Kumar et al., 2018). However, based on this model we expected impaired or abolished perinuclear clustering in plant lines unable to recognize XopQ (roql) or to initiate TNL immunity (edsl, $\left.n r g 1, n r g 1 \_a d r l\right)$. Despite, having reduced numbers of ETI-associated stromules, the plastid clustering still occurred when XopQ:mOrange2 was expressed in respective mutant backgrounds. Notably, plastid clustering was more pronounced in the mutants compared to

wild type. In contrast, perinuclear clustering in response to XopQ:mOrange2 expression was not slightly reduced in $n r g l$ plants (Fig. 7 A-K). In summary, we observe a negative correlation between stromule frequency and perinuclear plastid clustering. Accordingly, in our assays ETI induction of stromules was associated with lower plastid clustering compared to when ETI was disabled (Fig. 7H). These data suggest that increased chloroplast guidance towards the nucleus via stromules does not facilitate ETI but might be due to XopQ virulence activity.

\section{Is induction of peri-nuclear plastid clustering a part of XopQ's function?}

455 Stronger plastid clustering observed in the absence of ROQ1, EDS1, ADR1_NRG1 suggests it 456 may represent a consequence of undisturbed XopQ activity (Fig. 7A and 8B). This observation 457 partially contradicts the suggestion that peri-nuclear plastid clustering supports ETI responses by 458 facilitating more efficient transfer of pro-defense signals from plastids to the nucleus (discussed in Exposito-Rodriguez et al., 2020). If the sole function of peri-nuclear plastids is to enhance ETI, 460 why should the bacteria facilitate peri-nuclear plastid clustering via XopQ in the absence of 461 effector recognition? Conversely, why suppress clustering when ETI is induced by XopQ? Our results suggest that clustering may serve multiple functions or is the consequence of several stimuli in plant pathogen interactions. In support of this hypothesis while plastid clustering in $N$. benthamiana occurs in response to ETI triggering stimuli (e.g. TMV-p50 and RPS2 recognition Kumar et al., 2018, Ding et al., 2019), it also occurs in response to pattern triggered immunity (PTI) stimuli (Pst DC3000 AhopQ1-1, flg22 and $\mathrm{H}_{2} \mathrm{O}_{2}$, Ding et al., 2019), which demonstrates that plastid clustering is not ETI specific. Additionally, plastid clustering is not restricted to plant 
468 microbe interactions and has been found to be important for plastid inheritance during cell

469 division (Sheahan et al., 2004 and 2020) and has been observed following the exposure of $N$.

470 benthamiana epidermis leaf cells to cytokinin (Erickson et al., 2014). In summary, although

471 plastid accumulation at the nucleus is linked to plant microbe interactions it is not exclusively so,

472 and may reflect one output resulting from changes to different cell physiological parameters (i.e.,

473 altered hormone or $\mathrm{H}_{2} \mathrm{O}_{2}$ levels). Currently, although we see that XopQ activity induces

474 clustering, the trigger for this phenotype remains enigmatic and it remains to be seen whether it is 475 of any benefit to $X c v$ during an infection.

\section{Conclusion}

477 We set out in this study to test two hypotheses about stromule function in the context of effector 478 triggered immunity. We provide here experimental evidence for a direct link for ETI-induced 479 stromule formation, supporting the hypothesis of Caplan et al. (2015), which suggests that 480 stromules play a specific role during ETI. Our findings therefore encourage the enquiry of the 481 nature of this specific role in future. In contrast to this, our results do not support the second 482 hypothesis, which suggested that stromules might be needed to guide plastid movement towards 483 the nucleus (Kumar et al., 2018), highlighting the fact that there is currently no mechanistic 484 explanation for peri-nuclear plastid accumulation and that for this phenomenon fundamental work 485 still has to be done. Finally, the obtained results in the $n r g l$ and $a d r 1 \_n r g 1$ mutants (cooperative 486 activity of NRG1 and ADR1 in ETI signaling) show the potential of stromules to function as a 487 quantifiable and sensitive readout for branches of ETI signaling, which result in currently used 488 assays only in hard to detect differences. 


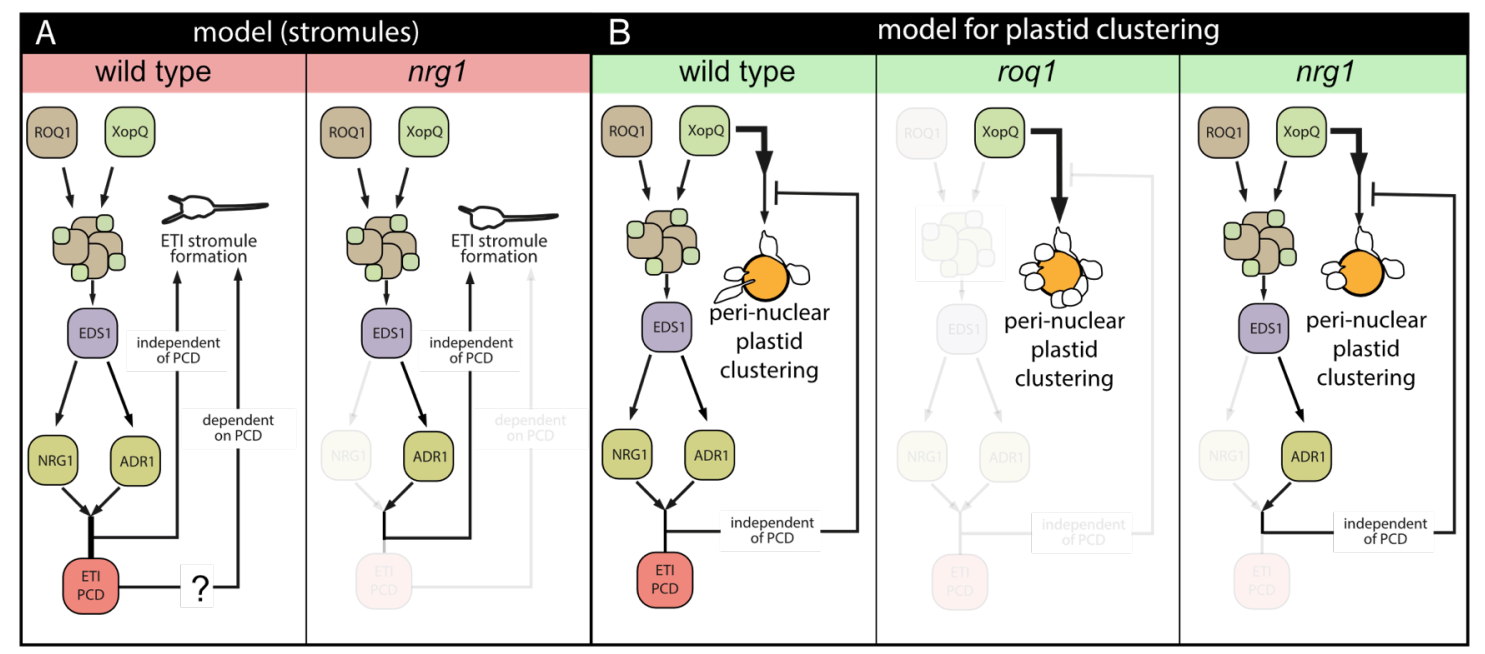

491 Fig. 8: Model of XopQ mediated stromule induction and peri-nuclear plastid clustering. (A)

492 Model for effector triggered immunity (ETI) induced stromule formation by XopQ in wild-type 493 plants (left panel): XopQ (Xanthomonas outer protein Q) is recognized by the TIR-NB-LRR (TOLL INTERLEUKIN 1 RECEPTOR NUCLEOTIDE BINDING LEUCIN RICH REPEAT) resistance protein ROQ1 (RECOCNITION OF XopQ 1), which forms with XopQ a heteromeric protein complex, the so called 'resistosome'. From the 'resistosome' the information of XopQ recognition is transferred with the help of EDS1 (ENHANCED DISEASE SUSCEPTIBILITY 1) to the RNL class helper NLRs NRG1 and ADR1, which culminates in PCD (programmed cell death) as well as stromule formation. In $n r g 1$ mutants (right panel) XopQ fails to induce cell death but at the same time still shows significant ETI stromule induction mediated by ADR1. This indicates the existence of a PCD independent ETI stromule induction pathway. However, at this point a role for PCD in stromule induction, or a role for stromules in PCD is not ruled out (?). (B) Model for peri-nuclear plastid clustering following $x o p Q$ expression: In wildtype plants (left) an intact ETI signal chain partially suppresses the strong peri-nuclear plastid clustering induced by XopQ presence. When ETI signal transduction is blocked (eds1, roq1 and adr_nrgl mutants) this suppression does not take place and plastid clustering is enhanced (middle). The restricted ETI signal chain in $n r g l$ mutants (right) is sufficient for suppression of induced peri-nuclear plastid clustering to wildtype levels, thus suppressing signals likely originate from this pathway. Both models only consider genes, which were analyzed as part of this study. The role of the different proteins forming distinct complexes with EDS1 will have to be elucidated in future. 


\section{Material and Methods}

\section{Plant material}

514 Nicotiana benthamiana plant lines used in this study were: wild type, roq1-3 and roq1-4 (Gantner 515 et al., 2019), eds1-1 (also referred to as eds1a-1; Ordon et al., 2017), nrg1-4 and nrg1-5 (Ordon et $516 a l ., 2021)$. An $a d r 1 \_n r g 1$ double mutant was created by genome editing using a derivative of 517 pDGE311, a plant transformation vector containing additional counter-selection markers and an 518 intron-optimized zCas $9 i$ gene, as recently described (Grützner et al., 2021, Stuttmann et al., 2021) 519 (Methods S1). For Xanthomonas inoculations transgenic $N$. benthamiana plants of the plant line 520 FNR:eGFP\#7-25 expressing the plastid marker FNR:eGFP (Schattat et al., 2011b) were used. 521 Plants were grown in long day conditions (16h day and 8h night) in green house chambers with 522 controlled temperature and humidity. Temperature was approximately $23{ }^{\circ} \mathrm{C}$ during the day and $52319^{\circ} \mathrm{C}$ at night. Relative humidity was kept around $55 \%$.

\section{Bacterial strains and cultivation}

525 E. coli Top10 cells were used for cloning and DNA propagation. Cells were cultivated at $37{ }^{\circ} \mathrm{C}$ in 526 LB with the appropriate antibiotic selection. Agrobacterium strain GV3101 (pMP90) (Koncz \& Schell, 1986) was grown in liquid or on solid YEB media containing rifampicin, gentamycin and either spectinomycin or carbenicillin, while $X c v$ strains were grown in NYG medium supplemented with rifampicin $\left(30^{\circ} \mathrm{C}\right.$ for both). Xanthomonas strains utilized were: $X c v 85-10$ 530 (wild-type; Thieme et al., 2005), Xcv $\Delta h r c N$ (strain deficient in an ATPase required for typeIII 531 secretion of effectors; Lorenz \& Büttner, 2009 and Xcv $\Delta x o p Q$ Adlung et al., 2016).

\section{Plasmids}

533 For visualization of plastids and stromules a plastid organelle marker construct was created using 534 the Modular Cloning Toolbox (Weber et al., 2011, Engler et al., 2014). The final construct 535 consisted of the $35 S$ promotor (pICH51277), the chloroplast transit peptide of RUBISCO (pAGS536 L0-\#115 in backbone pICH41258), eGFP (pAGS-L0-\#050 backbone pICH41264) and the NOS 537 terminator (pICH41421), assembled in an Level 1 acceptor plasmid. The XopQ:mOrange2 538 expression construct was described previously (Erickson et al., 2018; for more details see 539 Methods S2). 
Plasmids were transformed into A. tumefaciens strain GV3101 (pMP90). For transient expression experiments, strains harboring the binary vectors were grown overnight in $5 \mathrm{ml}$ YEB liquid cultures (with appropriate antibiotics), harvested by centrifugation and resuspended in agrobacterium infiltration medium $(10 \mathrm{mM} \mathrm{MgCl} 2 ; 5 \mathrm{mM}$ MES pH 5.6; $0.15 \mu \mathrm{M}$

Acetosyringone) with a final optical density (OD600nm) of 0.2. Bacteria harboring the plastid marker and the effector or the mORANGE2 control were mixed in a 1:1 ratio. Using a needless syringe, bacterial suspensions were inoculated into intercostal areas of the youngest fully expanded leaves of 5-6-week-old N. benthamiana plants (see Methods S3).

\section{Xanthomonas campestris pv. vesicatoria inoculations}

$X c v$ NYG liquid cultures were centrifuged to harvest cells, bacteria was resuspended in $10 \mathrm{mM}$ $\mathrm{MgCl}_{2}$, and suspensions adjusted to an OD600nm of 0.1. All three strains, as well as a buffer control, were then inoculated as described for A.tumefaciens. Plastids/stromules were observed at 2 days post-infiltration (dpi) using an epi-fluorescence microscope.

\section{Imaging hardware}

555 For image acquisition, an epi-fluorescence microscope (AxioObserver Z1) setup from Zeiss 556 (Jena, Germany) equipped with an X-Cite fluorescence light source and a MRm monochrome 557 camera (Zeiss, Jena, Germany) was used. GFP fluorescence was recorded using a 38 HE filter 558 cube (Carl Zeiss AG, Jena, Germany). mORANGE2 fluorescence was recorded utilizing the 43 559 HE filter cube (Carl Zeiss AG. Jena, Germany). The microscope manufacturer's software 560 (ZenBlue, Zeiss, Germany) controlled image acquisition. All images were captured using a 40x / 0.75 NA EC PLAN NEOFLUAR lens.

\section{Imaging procedures and image processing}

563 For quantification of stromule frequencies, a single leaf disc of each infiltration spot was 564 harvested using a cork borer. Leaf discs were vacuum-infiltrated, mounted on glass slides and 565 three independent z-stacks of the lower epidermis were collected in transmitted light, eGFP and 566 mORANGE2 channels. In order to obtain 2D extended depth of field images for quantification, 567 single images of the z-series of each channel were first exported into separate file folders and 568 subsequently combined into single images using software and procedures described in Schattat \& 569 Klösgen, 2009 (total of 3 images per disc). 
570 For quantification of stromule frequencies (SF\%) we measured the proportion of plastids with at

571 least one stromule (Erickson et al., 2014). To facilitate the faster quantification of stromule and

572 plastid counts in $N$. benthamiana tissues, we expanded on the previously published

573 MTBCellCounter (Franke et al., 2015) via a ridge detection-based stromule detection algorithm

574 (Möller \& Schattat, 2019). The extended MTBCellCounter allows for the detection of plastid

575 bodies as described in Franke et al., 2015 and identifies subsequently plastids with stromules.

576 The plastid nucleus association index (PNAI) was described previously (Erickson et al., 2014),

577 and represents the absolute number of plastids in close association with a given nucleus. PNAI

578 was evaluated in the 2D projected images (see image processing). Nuclei were counted as nucleus

579 associated when either the plastid body touched, overlapped with, or was within a distance of

$5804 \mu \mathrm{m}$ from the nucleus. $4 \mu \mathrm{m}$ corresponds to the average epidermis plastid diameter.

581 For sample sizes and details on statistical analysis of SF\% and PNAI see Supplemental materials

582 Methods S4 Notes S1.

583 Naming conventions

584 For conventions used to name mutants, genes, proteins and artificial DNA constructs see Methods 585 S5.

\section{Acknowledgments}

587 All Xcv strains were kindly provided by Prof. Ulla Bonas, Martin-Luther-Universität Halle588 Wittenberg, Germany. Dr. Sebastian Schornack, TSL, Cambridge, UK for advice on the 589 manuscript. This study was funded by the Deutsche Forschungsgemeinschaft (DFG, German 590 Research Foundation) - 400681449/GRK2498, STU642/1-1 and SFB-1403-414786233 as well 591 as Martin-Luther-University core funding. 


\section{References}

594

595

596

597

598

599

600

601

602

603

604

605

606

607

608

609

610

611

612

613

614

615

616

Adlung N, Bonas U (2017) Dissecting virulence function from recognition: cell death suppression in Nicotiana benthamiana by XopQ/HopQ1-family effectors relies on EDS1-dependent immunity. Plant J 91: 430-442

Adlung N, Prochaska H, Thieme S, Banik A, Blüher D, John P, Nagel O, Schulze S, Gantner J, Delker C, et al (2016) Non-host Resistance Induced by the Xanthomonas Effector XopQ Is Widespread within the Genus Nicotiana and Functionally Depends on EDS1. Front Plant Sci 7: 1796

Bi G, Su M, Li N, Liang Y, Dang S, Xu J, Hu M, Wang J, Zou M, Deng Y, et al (2021) The ZAR1 resistosome is a calcium-permeable channel triggering plant immune signaling. Cell 184: 3528-3541.e12

Bonardi V, Tang S, Stallmann A, Roberts M, Cherkis K, Dangl JL (2011) Expanded functions for a family of plant intracellular immune receptors beyond specific recognition of pathogen effectors. Proceedings of the National Academy of Sciences 108: 16463-16468

Büttner D (2016) Behind the lines-actions of bacterial type III effector proteins in plant cells. FEMS Microbiology Reviews 40: 894-937

Caplan JL, Kumar AS, Park E, Padmanabhan MS, Hoban K, Modla S, Czymmek K, Dinesh-Kumar SP (2015) Chloroplast Stromules Function during Innate Immunity. Developmental Cell 34: 45-57

Castel B, Ngou P-M, Cevik V, Redkar A, Kim D-S, Yang Y, Ding P, Jones JDG (2019) Diverse NLR immune receptors activate defence via the RPW8-NLR NRG1. New Phytologist 222: 966-980

Collier SM, Hamel L-P, Moffett P (2011) Cell death mediated by the N-terminal domains of a unique and highly conserved class of NB-LRR protein. MPMI 24: 918-931

Cui H, Tsuda K, Parker JE (2015) Effector-triggered immunity: from pathogen perception to robust defense. Annu Rev Plant Biol 66: 487-511

Delfosse K, Wozny MR, Jaipargas E-A, Barton KA, Anderson C, Mathur J (2016) Fluorescent Protein Aided Insights on Plastids and their Extensions: A Critical Appraisal. Front Plant Sci. doi: 10.3389/fpls.2015.01253 
Ding X, Jimenez-Gongora T, Krenz B, Lozano-Duran R (2019) Chloroplast clustering around the nucleus is a general response to pathogen perception in Nicotiana benthamiana. Mol Plant Pathol 8: 521

Engler C, Youles M, Gruetzner R, Ehnert T-M, Werner S, Jones JDG, Patron NJ, Marillonnet S (2014) A golden gate modular cloning toolbox for plants. ACS Synth Biol 3: 839-843

Erickson JL, Adlung N, Lampe C, Bonas U, Schattat MH (2018) The Xanthomonas effector XopL uncovers the role of microtubules in stromule extension and dynamics in Nicotiana benthamiana. Plant J 93: 856-870

Erickson JL, Ziegler J, Guevara D, Abel S, Klösgen RB, Mathur J, Rothstein SJ, Schattat MH (2014) Agrobacterium-derived cytokinin influences plastid morphology and starch accumulation in Nicotiana benthamiana during transient assays. BMC Plant Biol 14: 127-120

Franke L, Erickson JL, Rödel D, Schröter D, Storbeck B, Möller B, Schattat MH (2015) The "MTB Cell Counter" a versatile tool for the semi-automated quantification of sub-cellular phenotypes in fluorescence microscopy images. A case study on plastids, nuclei and peroxisomes. 26: 031-042

Gantner J, Ordon J, Kretschmer C, Guerois R, Stuttmann J (2019) An EDS1-SAG101 Complex Is Essential for TNL-Mediated Immunity in Nicotiana benthamiana. The Plant Cell 31: $2456-2474$

Gray JC, Hansen MR, Shaw DJ, Graham K, Dale R, Smallman P, Natesan SKA, Newell CA (2012) Plastid stromules are induced by stress treatments acting through abscisic acid. Plant J 69: $387-398$

Gray JC, Sullivan JA, Hibberd JM, Hansen MR (2001) Stromules: Mobile protrusions and interconnections between plastids. Plant Biology 3: 223-233

Grützner R, Martin P, Horn C, Mortensen S, Cram EJ, Lee-Parsons CWT, Stuttmann J, Marillonnet S (2021) High-efficiency genome editing in plants mediated by a Cas9 gene containing multiple introns. Plant Communications 2: 100135

Gunning BES (2005) Plastid stromules: video microscopy of their outgrowth, retraction, tensioning, anchoring, branching, bridging, and tip-shedding. Protoplasma 225: 33-42 
Holzinger A, Kwok EY, Hanson MR (2008) Effects of $\operatorname{arc3}$, arc5 and $\operatorname{arc} 6$ mutations on plastid morphology and stromule formation in green and nongreen tissues of Arabidopsis thaliana. Photochem Photobiol 84: 1324-1335

Jacob P, Kim NH, Wu F, Kasmi El F, Chi Y, Walton WG, Furzer OJ, Lietzan AD, Sunil S, Kempthorn K, et al (2021) Plant "helper" immune receptors are Ca2+-permeable nonselective cation channels. Science (New York, NY) 373: 420-425

Jubic LM, Saile S, Furzer OJ, Kasmi El F, Dangl JL (2019) Help wanted: helper NLRs and plant immune responses. Current Opinion in Plant Biology 50: 82-94

Koncz C, Schell J (1986) The promoter of T L-DNA gene 5 controls the tissue-specific expression of chimaeric genes carried by a novel type of Agrobacterium binary vector. 204: 383-396

Köhler RH, Hanson MR (2000) Plastid tubules of higher plants are tissue-specific and developmentally regulated. Journal of Cell Science 113 ( Pt 1): 81-89

Krenz B, Jeske H, Kleinow T (2012) The induction of stromule formation by a plant DNA-virus in epidermal leaf tissues suggests a novel intra- and intercellular macromolecular trafficking route. Front Plant Sci 3: 291

Kretschmer M, Damoo D, Djamei A, Kronstad J (2020) Chloroplasts and Plant Immunity: Where Are the Fungal Effectors? Pathogens 2020, Vol 9, Page 19 9: 19

\section{Kumar AS, Park E, Nedo A, Alqarni A, Ren L, Hoban K, Modla S, McDonald JH,} Kambhamettu C, Dinesh-Kumar SP, et al (2018) Stromule extension along microtubules coordinated with actin-mediated anchoring guides perinuclear chloroplast movement during innate immunity. Elife. doi: 10.7554/eLife.23625

Lapin D, Bhandari DD, Parker JE (2020) Origins and Immunity Networking Functions of EDS1 Family Proteins. Annu Rev Phytopathol 58: 253-276

Lapin D, Kovacova V, Sun X, Dongus JA, Bhandari D, Born von P, Bautor J, Guarneri N, Rzemieniewski J, Stuttmann J, et al (2019) A Coevolved EDS1-SAG101-NRG1 Module Mediates Cell Death Signaling by TIR-Domain Immune Receptors. The Plant Cell 31: 2430 2455 
Lorenz C, Büttner D (2009) Functional characterization of the type III secretion ATPase HrcN from the plant pathogen Xanthomonas campestris pv. vesicatoria. J Bacteriol 191: 1414-1428

Lu Y, Tsuda K (2021) Intimate Association of PRR- and NLR-Mediated Signaling in Plant Immunity. MPMI 34: 3-14

Ma S, Lapin D, Liu L, Sun Y, Song W, Zhang X, Logemann E, Yu D, Wang J, Jirschitzka J, et al (2020) Direct pathogen-induced assembly of an NLR immune receptor complex to form a holoenzyme. Science (New York, NY). doi: 10.1126/science.abe3069

Martin R, Qi T, Zhang H, Liu F, King M, Toth C, Nogales E, Staskawicz BJ (2020) Structure of the activated ROQ1 resistosome directly recognizing the pathogen effector XopQ. Science (New York, NY). doi: 10.1126/science.abd9993

Mathur J, Mammone A, Barton KA (2012) Organelle extensions in plant cells. Journal of Integrative Plant Biology 54: 851-867

Möller B, Schattat MH, 2019, 2019 Quantification of Stromule Frequencies in Microscope Images of Plastids Combining Ridge Detection and Geometric Criteria. pdfs.semanticscholar.org

Mullineaux PM, Selga T, Selga M, Exposito-Rodriguez M, Laissue PP, Gobiṇš V, Smirnoff N, Laser AO, 2010, Park E (2020) Spatial chloroplast-to-nucleus signalling involving plastid-nuclear complexes and stromules. Philos Trans R Soc Lond B Biol Sci 375: 20190405

Ordon J, Gantner J, Kemna J, Schwalgun L, Reschke M, Streubel J, Boch J, Stuttmann J (2017) Generation of chromosomal deletions in dicotyledonous plants employing a userfriendly genome editing toolkit. Plant J 89: 155-168

Ordon J, Martin P, Erickson JL, Ferik F, Balcke G, Bonas U, Stuttmann J (2021) Disentangling cause and consequence: genetic dissection of the DANGEROUS MIX2 risk locus, and activation of the DM2h NLR in autoimmunity. Plant J 106: 1008-1023

\section{Pruitt RN, Locci F, Wanke F, Zhang L, Saile SC, Joe A, Karelina D, Hua C, Fröhlich K,} Wan W-L, et al (2021) The EDS1-PAD4-ADR1 node mediates Arabidopsis patterntriggered immunity. Nature 1985 313:6005 598: 495-499 


\section{Qi T, Seong K, Thomazella DPT, Kim JR, Pham J, Seo E, Cho M-J, Schultink A,} Staskawicz BJ (2018) NRG1 functions downstream of EDS1 to regulate TIR-NLR-mediated plant immunity in Nicotiana benthamiana. Proc Natl Acad Sci USA 115: E10979-E10987

Saile SC, Jacob P, Castel B, Jubic LM, Salas-Gonzáles I, Bäcker M, Jones JDG, Dangl JL, Kasmi EI F (2020) Two unequally redundant "helper" immune receptor families mediate Arabidopsis thaliana intracellular "sensor" immune receptor functions. PLOS Biology 18: e3000783

Schattat M, Barton K, Baudisch B, Klösgen RB, Mathur J (2011) Plastid stromule branching

Schattat MH, Klösgen RB (2009) Improvement of plant cell microscope images by use of “depth of field-"extending software. Endocytobiosis and Cell Research 11-19

Schattat MH, Klösgen RB (2011) Induction of stromule formation by extracellular sucrose and glucose in epidermal leaf tissue of Arabidopsis thaliana. BMC Plant Biol 11: 115

Schultink A, Qi T, Lee A, Steinbrenner AD, Staskawicz B (2017) Roq1 mediates recognition of the Xanthomonas and Pseudomonas effector proteins XopQ and HopQ1. Plant J 92: 787795

Sheahan MB, Collings DA, Rose RJ, McCurdy DW (2020) ACTIN7 Is Required for Perinuclear Clustering of Chloroplasts during Arabidopsis Protoplast Culture. Plants 2020, Vol 9, Page 225 9: 225

Sheahan MB, Rose RJ, McCurdy DW (2004) Organelle inheritance in plant cell division: the actin cytoskeleton is required for unbiased inheritance of chloroplasts, mitochondria and endoplasmic reticulum in dividing protoplasts. Plant J 37: 379-390

Stuttmann J, Barthel K, Martin P, Ordon J, Erickson JL, Herr R, Ferik F, Kretschmer C, Berner T, Keilwagen J, et al (2021) Highly efficient multiplex editing: one-shot generation of $8 \times$ Nicotiana benthamiana and $12 \times$ Arabidopsis mutants. Plant J 106: 8-22 Blanvillain-Baufumé S, Harzen A, Bautor J, et al (2021) Pathogen effector recognitiondependent association of NRG1 with EDS1 and SAG101 in TNL receptor immunity. Nature Communications 12: 3335-15 
Sun Y, Zhu Y-X, Balint-Kurti PJ, Wang G-F (2020) Fine-Tuning Immunity: Players and Regulators for Plant NLRs. Trends in Plant Science 25: 695-713

Teper D, Burstein D, Salomon D, Gershovitz M, Pupko T, Sessa G (2016) Identification of novel Xanthomonas euvesicatoria type III effector proteins by a machine-learning approach. Mol Plant Pathol 17: 398-411

\section{Thieme F, Koebnik R, Bekel T, Berger C, Boch J, Büttner D, Caldana C, Gaigalat L,} Goesmann A, Kay S, et al (2005) Insights into genome plasticity and pathogenicity of the plant pathogenic bacterium Xanthomonas campestris pv. vesicatoria revealed by the complete genome sequence. J Bacteriol 187: 7254-7266

Tian H, Wu Z, Chen S, Ao K, Huang W, Yaghmaiean H, Sun T, Xu F, Zhang Y, Wang S, et al (2021) Activation of TIR signalling boosts pattern-triggered immunity. Nature 1985 313:6005 598: 500-+

Toruño TY, Stergiopoulos I, Coaker G (2016) Plant-Pathogen Effectors: Cellular Probes Interfering with Plant Defenses in Spatial and Temporal Manners. Annu Rev Phytopathol 54: $419-441$

Vismans G, van der Meer T, Langevoort O, Schreuder M, Bouwmeester H, Peisker H, Dörman P, Ketelaar T, van der Krol A (2016) Low-Phosphate Induction of Plastidal Stromules Is Dependent on Strigolactones But Not on the Canonical Strigolactone Signaling Component MAX2. Plant Physiol 172: 2235-2244

Wagner S, Stuttmann J, Rietz S, Guerois R, Brunstein E, Bautor J, Niefind K, Parker JE (2013) Structural basis for signaling by exclusive EDS1 heteromeric complexes with SAG101 or PAD4 in plant innate immunity. Cell Host Microbe 14: 619-630

Wang J, Hu M, Wang J, Qi J, Han Z, Wang G, Qi Y, Wang H-W, Zhou J-M, Chai J (2019a) Reconstitution and structure of a plant NLR resistosome conferring immunity. Science (New York, NY). doi: 10.1126/science.aav5870

Wang J, Wang J, Hu M, Wu S, Qi J, Wang G, Han Z, Qi Y, Gao N, Wang H-W, et al (2019b) Ligand-triggered allosteric ADP release primes a plant NLR complex. Science (New York, NY). doi: 10.1126/science.aav5868

Weber E, Engler C, Gruetzner R, Werner S, Marillonnet S (2011) A Modular Cloning System for Standardized Assembly of Multigene Constructs. PLoS ONE 6: e16765 


\section{Wu Z, Li M, Dong OX, Xia S, Liang W, Bao Y, Wasteneys G, Li X (2019) Differential} regulation of TNL-mediated immune signaling by redundant helper CNLs. New Phytologist 222: 938-953

Yuan M, Ngou BPM, Ding P, Xin X-F (2021) PTI-ETI crosstalk: an integrative view of plant immunity. Curr Opin Plant Biol 62: 102030 